INPLASY

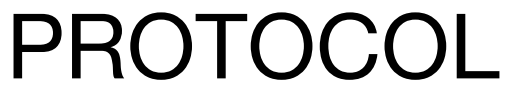

To cite: Li et al. Predictors for short-term successful weaning from continuous renal replacement therapy: a systematic review and metaanalysis. Inplasy protocol 202180082. doi: 10.37766/inplasy2021.8.0082

Received: 21 August 2021

Published: 21 August 2021

Corresponding author:

Yu Li

isleeyu@stu.xmu.edu.cn

Author Affiliation:

Department of Nephrology, Zhongshan Hospital affiliated to Xiamen University.

Support: Applicable.

Review Stage at time of this submission: The review has not yet started.

Conflicts of interest: None declared.

\section{Predictors for short-term successful weaning from continuous renal replacement therapy: a systematic review and meta-analysis}

Li, Y1; Feng, J2; Xu, B33; Deng, X4; Chen, Y5; Zhan, Y6; Lv, L7; Ye, Q8; Guo, X9; Guan, T10.

Review question / Objective: Continuous renal replacement therapy is a dialysis modality frequently used to treat patients with severe acute kidney injury, but there is limited information on when and how to stop CRRT in recovering patients for clinicians. This meta-analysis and systemic review aimed to further identify the predictors for short-term weaning from continuous renal replacement therapy.

Condition being studied: Acute kidney injury is a common complication found in critically ill patients. Although the individual risk varies widely depending on the underlying disease, the morbidity and mortality rate are very high in the intensive care unit. At present, clinicians practice continuous renal replacement therapy in very different ways, and there are some data regarding optimal commencement and dosing of continuous renal replacement therapy. However, less is known about the weaning criteria for continuous renal replacement therapy. Our object was to conduct a systemic review and meta-analysis to identify predictors for successful weaning from continuous renal replacement therapy among critically ill patients with acute kidney injury.

INPLASY registration number: This protocol was registered with the International Platform of Registered Systematic Review and Meta-Analysis Protocols (INPLASY) on 21 August 2021 and was last updated on 21 August 2021 (registration number INPLASY202180082).

\section{INTRODUCTION}

Review question / Objective: Continuous renal replacement therapy is a dialysis modality frequently used to treat patients with severe acute kidney injury, but there is limited information on when and how to stop CRRT in recovering patients for clinicians. This meta-analysis and systemic review aimed to further identify the predictors for short-term weaning from continuous renal replacement therapy. 
Condition being studied: Acute kidney injury is a common complication found in critically ill patients. Although the individual risk varies widely depending on the underlying disease, the morbidity and mortality rate are very high in the intensive care unit. At present, clinicians practice continuous renal replacement therapy in very different ways, and there are some data regarding optimal commencement and dosing of continuous renal replacement therapy. However, less is known about the weaning criteria for continuous renal replacement therapy. Our object was to conduct a systemic review and meta-analysis to identify predictors for successful weaning from continuous renal replacement therapy among critically ill patients with acute kidney injury.

\section{METHODS}

Search strategy: We conducted a literature search of studies through PubMed, Embase, and Cochrane Library. In addition, review articles on the topic will be searched for eligible articles. The search used the terms "continuous renal replacement therapy", "weaning", "discontinuation", "cessation", "factor", and "predictor" in several combinations. No restrictions were applied for time, language, or publication status.

Participant or population: Inclusion criteria: Patients with acute kidney injury requiring continuous renal replacement. Exclusion criteria: not being critically ill or in the ICU, end-stage renal disease on dialysis, peritoneal dialysis modality.

Intervention: Demographics, clinical and biochemical parameters that predicted the short-term successful weaning from continuous renal replacement therapy.

Comparator: Patients that failed weaning from the continuous renal replacement therapy.

Study designs to be included: Populationbased studies including case-control studies, prospective and retrospective cohort studies, or cross-sectional studies.
Eligibility criteria: (1) the study that reported predictive factors for short-term successful weaning from CRRT in critically ill patients with AKI in all countries and regions was included; (2) patients treated with CRRT for at least $24 \mathrm{~h}$ were divided into "success" and "failure" group according to their renal replacement therapy requirement in the short term after cessation of CRRT; and (3) the CRRT cycle had to be actually terminated (CRRT device disassembled) and not simply temporarily interrupted, e.g. for diagnostic or therapeutic measures outside the intensive care unit.

Information sources: We searched PubMed, Embase, and Cochrane Library for studies that reported predictive factors for successful weaning from CRRT from database inception to August 2021 without language restrictions. The references of the retrieved articles were also reviewed for additional reports to avoid missing out on our search.

Main outcome(s): Successful weaning from continuous renal replacement therapy.

Data management: Data extraction will be independently screened by two reviewers. Any conflict will be identified and resolved through discussion and, if necessary, with a third reviewer. The following data will be collected using a standardized data table: author, year, country, number of patients, type of study, the definition for "successful weaning from continuous renal replacement therapy" and predictive factors.

Quality assessment / Risk of bias analysis: Quality Assessment Tools for observational studies will be used to assess. The judgment of quality will be assessed by two authors independently, and any discrepancies will be resolved by discussion, or with the involvement of a third reviewer when necessary.

Strategy of data synthesis: Stata $\mathbf{1 6 . 0}$ software to realize data conversion and effect size combination. A quantitative summation will be done for predictive 
factors when an adequate number of similar factors are available. Publication bias will be assessed by examining funnel plot asymmetry and Egger's test. A fixedeffect model will be applied if the results are homogeneous; if the results are heterogeneous $\left(I^{2}>50 \%\right)$, a random-effects model will be applied.

Subgroup analysis: Any available subgroup analysis for a predictive factor will be critically evaluated and presented.

Sensitivity analysis: Exclude each study included in the analysis one by one, reanalyze and summarize the data, and compare the differences between the retrieved results and the original results. Therefore, we will be able to discover the impact of individual studies on overall results and whether the results are reliable.

Country(ies) involved: China.

Keywords: Continuous renal replacement therapy; acute kidney injury; weaning; predictors; meta-analysis.

Contributions of each author:

Author 1 - Yu Li.

Email: isleeyu@stu.xmu.edu.cn

Author 2 - Jiaxing Feng.

Email: jiaxingfeng05@gmail.com

Author 3 - Bo Xu.

Author 4 - Xiaoqi Deng.

Author 5 - Yulei Chen.

Author 6 - Ying Zhan.

Author 7 - Liangchen Lv.

Author 8 - Qing Ye.

Author 9 - Xiaodan Guo.

Author 10 - Tianjun Guan.

Email: guantianjun@aliyun.com 